\title{
Contrast-enhanced CT longitudinal tail sign as a marker of positive resection margins in adenoid cystic carcinoma of the central airway
}

\author{
Shu-Chao Wang ${ }^{1 \#}$, Le-Kang Yin ${ }^{2 \#}$, Yu Zhang ${ }^{2}$, Li-Min Xue ${ }^{1,3}$, Jian-Ding Ye ${ }^{2}$, Guang-Yu Tao ${ }^{2}$ Hong Yu ${ }^{2}$, \\ Jin-Wei Qiang ${ }^{1}$
}

${ }^{1}$ Department of Radiology, Jinshan Hospital, Fudan University, Shanghai, China; ${ }^{2}$ Department of Radiology, Shanghai Chest Hospital, Shanghai Jiao Tong University, Shanghai, China; ${ }^{3}$ Shanghai Institute of Imaging Medicine, Fudan University, Shanghai, China

Contributions: (I) Conception and design: JW Qiang; (II) Administrative support: JW Qiang, H Yu; (III) Provision of study materials or patients: LK Yin; (IV) Collection and assembly of data: SC Wang; (V) Data analysis and interpretation: SC Wang, JW Qiang; (VI) Manuscript writing: All authors; (VII) Final approval of manuscript: All authors.

\#These authors contributed equally to this work.

Correspondence to: Jin-Wei Qiang, MD, PhD. Department of Radiology, Jinshan Hospital, Fudan University, 1508 Longhang Road, Shanghai 201508, China. Email: dr.jinweiqiang@163.com; Hong Yu, MD, PhD. Department of Radiology, Shanghai Chest Hospital, Shanghai Jiao Tong University, 241 West Huaihai Road, Shanghai 200030, China. Email: yuhongphd@163.com.

\begin{abstract}
Background: Due to submucosal infiltration's biological nature along the airway, adenoid cystic carcinoma (ACC) frequently leaves positive surgical margins. This study evaluated the clinicopathologic, and computed tomography (CT) features for predicting surgical margin status in central airway ACC.

Methods: We retrospectively analyzed the files of 71 patients with ACC of the central airway proven by histopathology and surgery who had presented between January 2010 and December 2018. All patients were classified into positive and negative surgical margin groups according to margin status. Univariate analysis and multivariable logistic regression models were then performed to compare demography, histopathology, and CT characteristics between ACC patients with positive and negative margins.

Results: After surgical resection, 59 (83.1\%) patients had positive margins, and 12 (16.9\%) had negative margins. The contrast-enhanced CT (CECT) longitudinal tail sign (LTS) was identified in 55 of 59 (93.2\%) patients with positive margins and was the only feature that had a significant association with positive margins (odds ratio 41.250, 95\% CI: 7.886-215.767; $\mathrm{P}<0.001$ ). Moreover, positive margins in upper or/and lower directions were associated with the LTS in corresponding directions $(\mathrm{P}<0.001)$.

Conclusions: Most central airway ACC patients exhibited positive margins following surgery. The appearance of the LTS on CECT was significantly associated with positive margins and could help preoperatively predict the submucosal invasion of ACC.
\end{abstract}

Keywords: adenoid cystic carcinoma (ACC); positive surgical margin; longitudinal tail sign (LTS); central airway; computed tomography (CT)

Submitted Sep 02, 2020. Accepted for publication Mar 18, 2021.

doi: $10.21037 /$ jtd-20-2929

View this article at: http://dx.doi.org/10.21037/jtd-20-2929

\section{Introduction}

Salivary gland-type tumors generally occur in the head and neck, but other locations such as the lungs, breasts, skin, and cervix have also been reported (1). Adenoid cystic carcinoma (ACC) is the most common type of salivary gland-type cancer in the central airway and is mainly found in patients in their 40s and 50s with equal distribution between the sexes. Histologically, ACC originates from the 
airway submucosal seromucous glands, with a tendency to submucosal invasion and perineural infiltration (2). Due to ACC's indolent but progressive growth pattern, early symptoms and signs are not common and lack specificity, which often leads to a delayed definite diagnosis and most patients presenting with locally advanced disease (3). To date, surgical resection has been the main treatment for ACC of the airway (4), and compared with other treatments, primary surgery can achieve long-term survival and even cure some patients. Complete resection with negative margins is considered to be the most important association with improved survival outcomes (5).

However, it is usually difficult to achieve tumor-free margins in patients with ACC of the central airway. As tumor cells spread in the airway submucosa, the microscopic level of tumor invasion often exceeds what is grossly apparent and involves a longer airway (6). Thus, preoperative bronchoscopy cannot accurately assess the longitudinal submucosa extent of tumor cells by sampling small tissues, and surgeons can only determine tumor size by gross inspection for resection but cannot recognize microscopic submucosal lesions (7). In cases where intraoperative frozen section pathology shows positive margins, surgeons may further resect residual lesions, as positive surgical margins might lead to a late regional recurrence and adversely impact survival outcomes (8). However, resection margins may remain positive even after additional resection, increasing anastomotic tension, leading to an unsafe anastomosis. For ACC patients with excessive microscopic involvement of the airway, the safety of anastomoses should be the main concern, and the airway resection length should be minimized when removing tumors (9).

A challenge that remains for surgeons is how to achieve a balance between margin status and anastomosis tension (2). Accurately predicting surgical margin status before surgery will enable surgeons to make a more appropriate treatment plan. For patients who are predicted to have positive margins before surgery, additional resections might be avoided, and for patients who are predicted to have negative margins, excessive airway resection may not be required. Computed tomography $(\mathrm{CT})$ is a convenient and non-invasive method for diagnosing airway ACC, and contrast-enhanced images can provide information about the blood supply of tumors. CT can display the airway lumen and the extramural and longitudinal involvements of tumors (10). However, due to ACC's rarity in the central airway, few previous studies have focused on the possible predictive factors for resection margin status. Therefore, this study aimed to investigate whether the characteristics of demography, histopathology, and $\mathrm{CT}$ in ACC could predict margin status, thereby guiding surgical decision-making and subsequent treatment.

We present the following article following the MADR checklist (available at http://dx.doi.org/10.21037/jtd-20-2929).

\section{Methods}

\section{Patients}

The study was conducted in accordance with the Declaration of Helsinki (as revised in 2013). The study was approved by the Ethics Committee of the Shanghai Chest Hospital (2019YNJCQ02) and individual consent for this retrospective analysis was waived. The picture archiving and communication system database was searched to identify patients who had been diagnosed with central airway diseases between January 1, 2010, and December 31, 2018, in the Shanghai Chest Hospital. Patients with pathologically confirmed ACC were subsequently selected for the study. All patients underwent primary surgery with or without adjuvant therapy, while no patients received neoadjuvant therapy. Of the 79 patients with ACC in the central airway, eight were excluded because of a lack of contrast-enhanced CT (CECT) scans. Thus, our final study cohort comprised 71 ACC patients who had preoperative CT scans no more than 2 weeks before surgery.

\section{Histopathological analysis}

Patients with tracheal ACC received tracheal or laryngotracheal resection and one-stage end-to-end anastomosis, while in those with main bronchial ACC, bronchial sleeve resection and pneumonectomy followed by one-stage end-to-end anastomosis were performed. Carinal resection with or without pulmonary resection was performed if the carina was involved. The transected trachea or bronchus was assessed for tumor malignancy, and the resection margin status based on the intraoperative frozen sections. A subset of patients underwent additional airway resections if a positive margin was found, although there were no macroscopically positive surgical margins in our patients. Tumor histopathology and final margin status (negative or positive margin) was diagnosed based on paraffin sections, and a positive margin was determined if there were microscopically residual lesions after surgical resection. Other histopathological characteristics of the tumors, including resected tracheal or bronchial length, tumor longitudinal and traverse length 

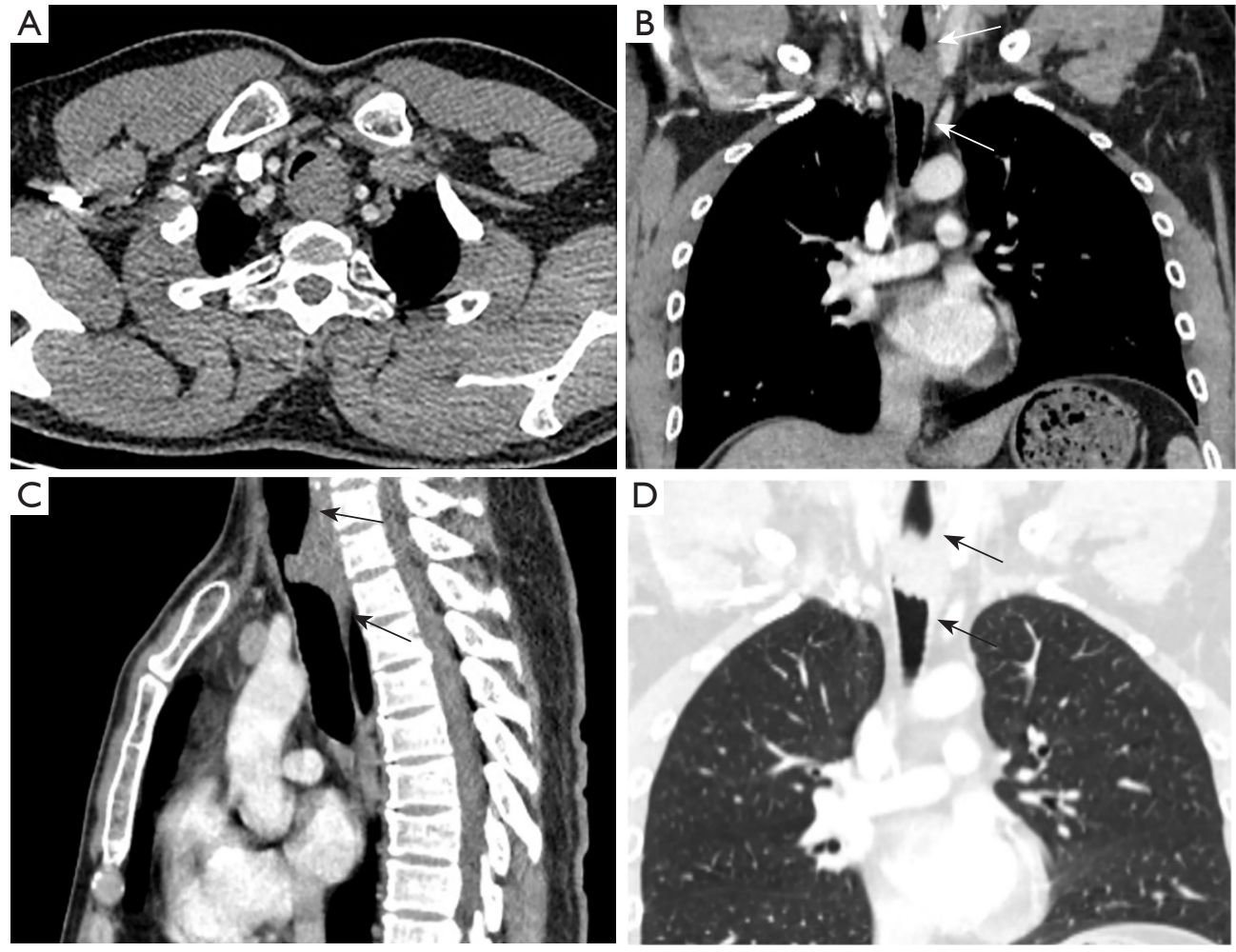

Figure 1 A 36-year-old man with adenoid cystic carcinoma (ACC). Axial chest contrast-enhanced CT (CECT) image (A) and reformatted sagittal and coronal images (B and $\mathrm{C}$ ) on the soft-tissue window show a broad base nodule with transmural growth involving the upper trachea (arrows). The longitudinal tail sign (LTS) was bilaterally identified at the left and rear walls of the trachea (arrows) and pathology confirmed bilateral positive margins. Coronal chest CECT image (D) on the lung window also shows a bilateral LTS.

and their ratio, regional lymph node metastasis (LNM), and invasion of surrounding tissues (fat, nerves, blood vessels, and thyroid), were also extracted.

\section{Imaging}

All 71 patients underwent both plain and CECT scans of the neck or chest using multislice CT scanners. CT was performed using a protocol with the following settings: $120 \mathrm{kV} ; 320 \mathrm{~mA}$; collimation, $0.6 \mathrm{~mm}$; slice thicknesses, 1$5 \mathrm{~mm}$. Nonionic contrast medium (1.5 mL/kg body weight) ioversol $[350 \mathrm{mgI} / \mathrm{mL}$, Hengrui Medicine, Lianyungang, China]) was administered via a median cubital vein at a rate of 3.0-4.0 mL/s with a 30- to 40-second delay. Coronal and sagittal images of both lung and soft-tissue windows were reconstructed based on the original axial-section data with corresponding section thickness.

Two radiologists reviewed the CT images, and any discrepancies were resolved by consensus. The CT findings, including the location, shape, growth mode, contour, border, homogeneity in the arterial phase, and longitudinal tail sign (LTS) of the primary tumor, were analyzed. Based on our observation and Goldsher et al.'s definition of "dural tail sign" (11), we ascribed the LTS based on the following characteristics: (I) a thickened airway wall tapering away from the tumor along the longitudinal axis of the airway, with a smooth inner surface and a length greater than 5 $\mathrm{mm}$; (II) presenting beyond the tumor on two or more consecutive axial sections; (III) presenting on two or more consecutive sagittal or coronal sections through the center of the tumor, and; (IV) exhibiting enhancement similar to that of the tumor mass itself (Figure 1).

\section{Statistical analysis}

All statistical analyses were performed using SPSS 23.0 software (IBM Corp, Chicago, IL, USA). Continuous variables were presented as mean \pm standard deviation 
Table 1 Clinicopathologic features in patients with ACC of the central airway

\begin{tabular}{|c|c|c|c|c|}
\hline Variables & \multicolumn{3}{|c|}{ No. of patients (\%) } & P-value \\
\hline Age (y), median (range) & $45(25-67)$ & $45(25-67)$ & $45(33-67)$ & 0.854 \\
\hline Gender & & & & 0.707 \\
\hline Female & $32(45.1)$ & $26(44.1)$ & $6(50.0)$ & \\
\hline Resected length (mm), median (range) & $30(10-70)$ & $30(10-60)$ & $22(12-70)$ & 0.469 \\
\hline Longitudinal length (mm), median (range) & $30(8-80)$ & $30(8-80)$ & $21(10-45)$ & $0.086^{\mathrm{a}}$ \\
\hline Traverse length $(\mathrm{mm})$, mean $\pm \mathrm{SD}$ & $21 \pm 9$ & $22 \pm 9$ & $19 \pm 10$ & 0.298 \\
\hline Longitudinal/Traverse ratio & & & & 0.250 \\
\hline Regional LNM & $9(12.7)$ & $9(15.3)$ & $0(0.0)$ & 0.331 \\
\hline Invasion of surrounding tissues & $16(22.5)$ & $15(25.4)$ & $1(8.3)$ & 0.361 \\
\hline
\end{tabular}

a, Statistically significant at $\mathrm{P}<0.1$. ACC, adenoid cystic carcinoma; R0, negative surgical margin; R1, positive surgical margin; LNM, lymph node metastasis.

or median and compared between patients with positive and negative surgical margins using two independent sample $t$-tests or Mann-Whitney $\mathrm{U}$ tests, as appropriate. Categorical variables were presented as count and percentage and compared using the Chi-square test or Fisher's exact test. A P-value $<0.1$ indicated statistical significance in the univariable analysis to avoid missing some important factors, and adjustments for multiple comparisons were made if needed by using Bonferroni correction. The positive and negative margins were taken as dependent variables, and the statistically significant variables obtained from the univariable analysis were treated as independent variables in the multivariable logistic regression analysis. A P-value of $<0.05$ was considered statistically significant in the multivariable logistic analysis. Section thickness was included in the logistic regression model as a possible confounding factor, considering different CT scan section thicknesses. All P-values were two-sided, and variables used to stratify margin status included clinicopathologic and CT characteristics of the tumors. The linear regression model was used to test whether independent variables entering the logistic regression model had multicollinearity. Moreover, multiple comparisons between surgical margin status and LTS on CECT were performed by Post hoc testing, with the absolute values of adjusted standardized residuals (ASR) $>2$ considered statistically significant.

\section{Results}

\section{Patients and tumor characteristics}

The clinicopathologic characteristics of ACC in the central airway stratified by margin status are shown in Table 1 . The 71 patients with ACC included 32 (45.1\%) women and $39(54.9 \%)$ men with a median age of 45 (range, 25-67), and there were no significant differences in sex and age between patients with positive and negative surgical margins. Positive margins were found in $83.1 \%$ (59 of 71) patients.

Patients with positive margins were more likely to have greater tumor longitudinal length than those with negative margins (median, 30 vs. $21 \mathrm{~mm} ; \mathrm{P}=0.086$ ), and most ACCs (63/71, 88.7\%) had greater longitudinal length than transverse length. The differences between patients with positive and negative margins were not statistically significant in the transverse length, longitudinal to transverse ratio, resected airway length, regional LNM, and invasion of surrounding tissues $(\mathrm{P}=0.298,0.250,0.469,0.331,0.361$, respectively). All nine patients with regional LNM and 15 of $16(93.4 \%)$ patients with invasion of surrounding tissues were found in the group of positive margins. The logistic regression analysis showed no significant difference in the longitudinal tumor diameter between the patients with positive and negative margins $(\mathrm{P}=0.822)$. 
Table 2 CT characteristics in patients with ACC of the central airway

\begin{tabular}{|c|c|c|c|c|}
\hline Variables & \multicolumn{3}{|c|}{ No. of patients (\%) } & $P$ value \\
\hline Location & & & & 0.537 \\
\hline Cervical trachea & $16(22.5)$ & $14(23.7)$ & $2(16.7)$ & \\
\hline Thoracic trachea & $36(50.7)$ & $31(52.5)$ & $5(41.6)$ & \\
\hline Main bronchus & $10(14.1)$ & $8(13.6)$ & $2(16.7)$ & \\
\hline Shape & & & & 0.608 \\
\hline Narrow base nodule & $1(1.4)$ & $1(1.7)$ & $0(0.0)$ & \\
\hline Broad base nodule & 39 (54.9) & $31(52.5)$ & $8(66.7)$ & \\
\hline Extraluminal & $2(2.8)$ & $1(1.7)$ & $1(8.3)$ & \\
\hline Transmural & $42(59.2)$ & $38(64.4)$ & $4(33.3)$ & \\
\hline Contour & & & & 0.631 \\
\hline Smooth & $46(64.8)$ & $37(62.7)$ & $9(75.0)$ & \\
\hline Lobular & $25(35.2)$ & $22(37.3)$ & $3(25.0)$ & \\
\hline Border & & & & 1.000 \\
\hline Well defined & $46(64.8)$ & $38(64.4)$ & $8(66.7)$ & \\
\hline Yes & $58(81.7)$ & 55 (93.2) & $3(25.0)$ & \\
\hline No & $13(18.3)$ & $4(6.8)$ & $9(75.0)$ & \\
\hline
\end{tabular}

${ }^{a}$, statistically significant at $\mathrm{P}<0.1$. CT, computed tomography; ACC, adenoid cystic carcinoma; R0, negative surgical margin; R1, positive surgical margin.

\section{CT findings}

CT characteristics stratified by margin status are shown in Table 2. Of the 71 ACCs, 42 (59.2\%) tumors appeared to grow transmurally, while $(27,38.0 \%)$ were growing endoluminally. Patients with transmural growth were more likely to have positive margins $(38 / 42,90.5 \%)$ than those with endoluminal growth $(20 / 27,74.1 \% ; \mathrm{P}=0.068)$. An LTS was seen on coronary or sagittal planar reformation CECT scans in 58 of $71(81.7 \%)$ patients, and this sign was more commonly observed in patients with positive margins
$(55 / 59,93.2 \%)$ than those with negative margins (3/12, $25.0 \% ; \mathrm{P}<0.001)$. ACCs mainly originated from the thoracic trachea $(36,50.7 \%)$ and appeared as a broad base nodule $(39,54.9 \%)$ or annular thickened wall $(31,43.7 \%)$ on CT axial-section images. Approximately two-thirds of ACCs appeared as a well-defined border and smooth contour, and half had a heterogeneous attenuation on CECT. No significant differences were found in the location, shape, border, contour, and attenuation between the two groups.

The logistic regression model evaluating potential 
Table 3 Potential markers of positive surgical margins in ACC of the central airway

\begin{tabular}{lccc}
\hline Variables & Odds ratio & $95 \% \mathrm{Cl}$ & $\mathrm{P}$ value \\
\hline Longitudinal tail sign $(\mathrm{ref=No})$ & 41.250 & $7.886,215.767$ & $<.001^{\mathrm{a}}$ \\
Longitudinal length $(\mathrm{mm})$ & - & - & 0.822 \\
Growth mode $(\mathrm{ref}=$ Intraluminal) & - & - & 0.309 \\
Extraluminal & - & - & 0.522 \\
Transmural & - & - & 0.230 \\
Section thickness $(\mathrm{ref}=1 \mathrm{~mm})$ & - & 0.884 & \\
\hline
\end{tabular}

${ }^{\mathrm{a}}$, statistically significant at $\mathrm{P}<0.05$. ACC, adenoid cystic carcinoma; $\mathrm{Cl}$, confidence interval; ref, reference level.

Table 4 Correlation between the LTS and surgical margin status with additional airway resections

\begin{tabular}{lccr}
\hline \multirow{2}{*}{ Variables } & \multicolumn{3}{c}{ No. of patients $(\%)$} \\
\cline { 2 - 4 } & Total $(\mathrm{n}=18)$ & Without LTS $(\mathrm{n}=1)$ & With LTS $(\mathrm{n}=17)$ \\
\hline R0 & $5(27.8)$ & $1(100.0)$ & $4(23.5)$ \\
R1 & $13(72.2)$ & $0(0.0)$ & $13(76.5)$ \\
\hline
\end{tabular}

LTS, longitudinal tail sign; R0, negative surgical margin; R1, positive surgical margin.

Table 5 Correlation of directions between surgical margin status and LTS on CECT

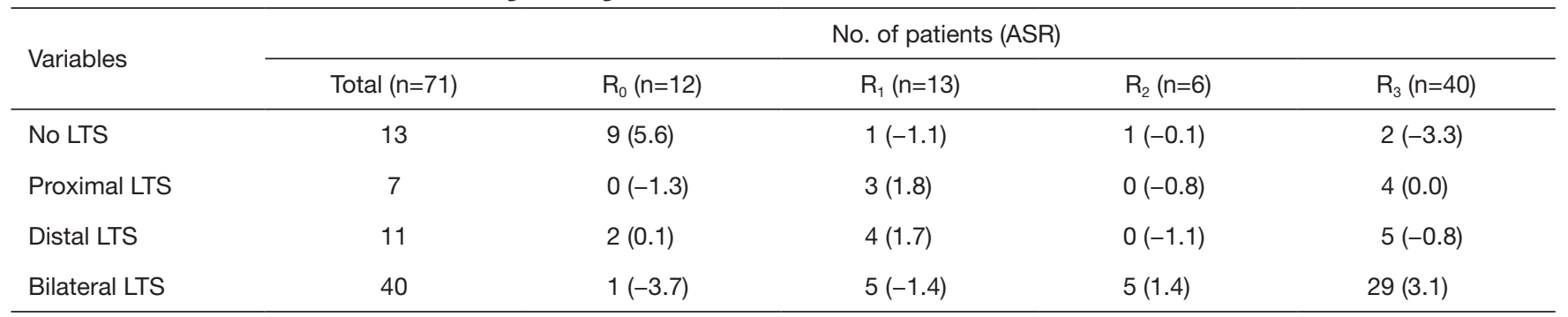

LTS, longitudinal tail sign; CECT, contrast-enhanced computed tomography; $A S R$, adjusted standardized residuals; $R_{0}$, negative margin;

$\mathrm{R}_{1}$, proximal positive margin; $\mathrm{R}_{2}$, distal positive margin; $\mathrm{R}_{3}$, Bilateral positive margins.

markers for positive surgical margins are shown in Table 3. Of the factors considered, only the LTS was an independent marker for positive surgical margins (odds ratio 41.250, 95\% CI: 7.886-215.767; $\mathrm{P}<0.001$ ), and its sensitivity, specificity, and accuracy were $93.2 \%, 75.0 \%$, and $90.1 \%$, respectively. Neither longitudinal length nor the growth mode of tumors on CT images had a significant difference $(\mathrm{P}=0.822$ and 0.309$)$, and different $\mathrm{CT}$ section thicknesses did not affect the observation of the relationship between the LTS and resection margins $(\mathrm{P}=0.884)$. Also, there was no multicollinearity in this model; that is, there was no significant correlation between the above factors.

Correlation between the LTS and surgical margin status with additional resection is shown in Table 4. While
$13(76.5 \%)$ patients with the LTS retained positive margins after additional resections, four patients $(23.5 \%)$ with an LTS turned into negative margins, although one died one month after surgery due to an anastomotic complication. Only one patient without an LTS obtained a negative margin after additional resection.

\section{CT-pathology correlation}

Correlation between surgical margin status and the LTS on CECT stratified by proximal, distal, and bilateral directions are shown in Table 5. CT-pathology correlation showed that positive margins in different directions tended to reveal an LTS in corresponding directions $(\mathrm{P}<0.001)$. Bilateral 

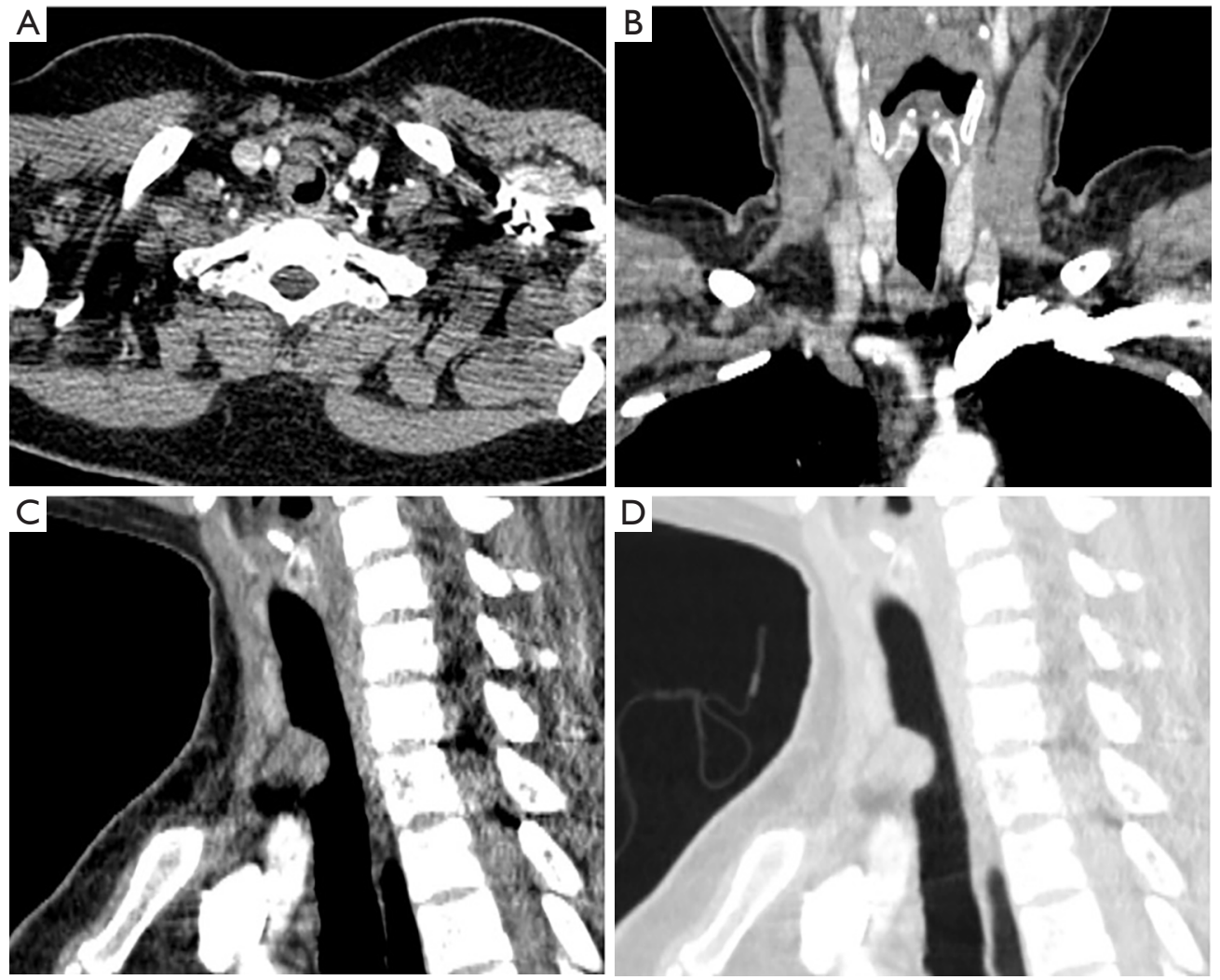

Figure 2 A 43-year-old woman with adenoid cystic carcinoma (ACC). Axial chest contrast-enhanced CT (CECT) image (A) and reformatted coronal and sagittal images (B and C) on the soft-tissue window show a broad base nodule with mild lobular shape involving the upper trachea. No longitudinal tail sign (LTS) was identified at either the proximal or distal side of the tumor and pathology conformed bilateral negative margins. Sagittal CECT image (D) on the lung window also shows no LTS in either direction.

positive margins tended to manifest a bilateral LTS rather than no LTS (ASR $=3.1,-3.3$ ), while bilateral negative margins were more likely to show no LTS rather than a bilateral LTS (ASR = 5.6, -3.7) (Figures 1,2).

\section{Discussion}

Due to the low incidence of ACC in the central airway, few reports have focused on factors related to surgical margins. Based on a large patient cohort in our institution, this study analyzed clinicopathologic and CT characteristics to identify their surgical margin status association. In ACC patients undergoing surgical resection, positive margins were found in most patients. Furthermore, this study was the first to propose the LTS on CECT, which was identified to be the only independent marker for positive surgical margins.

Tumor-free resection is hard to achieve for ACC with extensive longitudinal involvement of the airway. More positive surgical margins ( $83.1 \%$ ) were observed in our cohort than other studies, which had approximately $50 \%$ positive margins $(6,12)$. Although additional resections were performed in some cases in our study, most remained positive $(72.2 \%)$. This may be because surgeons are usually uncertain about the degree of microscopical tumor invasion on the airway's longitudinal axis before and during surgery.

As a potential CT characteristic first proposed by us, the LTS appeared in most patients in this study who had positive margins (93.2\%). Moreover, the direction of the LTS on the longitudinal axis of the airway corresponded to that of positive surgical margins. This could indicate ACC's unique biological behavior, which sees tumor cells tending to infiltrate in the submucosa along the airway. The longitudinal tail gradually tapered from the tumor body to the distal end, reflecting tumor cells slowly but progressively growing away from the tumor body. Also, the longitudinal tail's smooth inner surface suggests the 
tumor had not invaded the mucous layer yet so could not be visible by bronchoscopy. The results of additional resections also suggested that for cases with LTS, even if the tracheal resection length were less than $6 \mathrm{~cm}$, it would be better not to remove it further as this would shorten the operation time and reduce the risk of postoperative anastomotic complications. Some studies have suggested that postoperative radiotherapy is effective in long-term survival for patients with positive margins $(2,5,13,14)$. The longitudinal tails were mildly enhanced, similar to the primary tumors, which also indicated that these tail components were histopathologically the same as the primary tumors.

Consistent with previous studies, most airway ACCs in our study developed in the trachea, followed by main bronchi $(15-17)$. These tumors had a striking tendency towards a submucosal extension that manifested on CT images mainly as broad base masses or circumferential wall thickening, causing a luminal obstruction $(10,17)$. No significant difference was found in tumor shape between the patients with and without positive margins, and the tumor shapes on the cross-section reflected the local infiltration between tracheal rings, rather than the longitudinal invasion (18). Consistent with Luo's study (10), more than half of ACCs had a transmural growth pattern, which was more likely to have positive surgical margins, whereas ACCs with intraluminal growth were less frequent and tended to be negative margins. The transmural growth pattern suggested a wide range of tumor invasion in all directions, including the longitudinal axis.

Due to the rarity of pulmonary ACC, few studies have investigated the factors related to positive margins. Yang et al. analyzed the clinicopathologic characteristics of 132 patients with tracheal ACC from the NCDB database presenting between 1998 to 2014 (6) and found that only the clinical T-status of T4 had a significant association with positive margins. Bhattacharyya et al. define T4 staging as the tracheal tumor spreading to adjacent organs or structures that may indicate tumors' significant local invasiveness (19). Although 15 of $16(94 \%)$ cases with invading surrounding tissues in our study had positive surgical margins, no significant difference was found between positive and negative margins. We believe that the low incidence of invading surrounding tissues explained this as tumors with longer longitudinal length tended to leave positive margins. However, there was no statistical significance in the subsequent multivariable analysis, which indicated that microscopic tumor extension should be a more important factor for positive margins compared to the gross tumor length measured by pathological size. Similar results were obtained in Yang and colleagues' study, but longitudinal and axial diameters were not analyzed separately in their study.

This study had several limitations. First, given the inherent biases of a retrospective study, the results should be interpreted with caution. Second, all CECT images were scanned only during the arterial phase in our institution. The enhanced characteristics of the LTS during the venous and delayed phases might provide more valuable information about the nature of different tails, which might explain why some ACCs with an LTS did not show positive surgical margins. Finally, we did not investigate whether other types of tumors in the central airway had an LTS on CT images and their differences from ACCs. Further large-scale and multi-center studies are needed to verify the indicative efficiency of the LTS for surgical margin status.

\section{Conclusions}

Surgical margin status was an important determinant of airway length for resection in patients with tumors. Most ACCs in the central airway were resected with positive surgical margins, and the appearance of an LTS on CECT was a significant marker for positive margins. Further knowledge of the correlation between the length of the longitudinal tail and the extent of microscopic invasion in the tail of airway ACCs may contribute to the therapeutic plan.

\section{Acknowledgments}

Funding: This work was supported by the National Natural Science Foundation of P. R. China (Nos. 81871353, 82071873); the Shanghai Municipal Health Commission (No. ZK2019B01); and the Shanghai Chest Hospital (No. 2019YNJCQ02).

\section{Footnote}

Reporting Checklist: All authors have completed the MDAR checklist. Available at http://dx.doi.org/10.21037/jtd-202929

Data Sharing Statement: Available at http://dx.doi. org/10.21037/jtd-20-2929

Conflicts of Interest: All authors have completed the ICMJE uniform disclosure form (available at http://dx.doi. org/10.21037/jtd-20-2929). The authors have no conflicts 
of interest to declare.

Ethical Statement: The authors are accountable for all aspects of the work in ensuring that questions related to the accuracy or integrity of any part of the work are appropriately investigated and resolved. The study was conducted in accordance with the Declaration of Helsinki (as revised in 2013). The study was approved by the Ethics Committee of the Shanghai Chest Hospital (2019YNJCQ02) and individual consent for this retrospective analysis was waived.

Open Access Statement: This is an Open Access article distributed in accordance with the Creative Commons Attribution-NonCommercial-NoDerivs 4.0 International License (CC BY-NC-ND 4.0), which permits the noncommercial replication and distribution of the article with the strict proviso that no changes or edits are made and the original work is properly cited (including links to both the formal publication through the relevant DOI and the license). See: https://creativecommons.org/licenses/by-nc-nd/4.0/.

\section{References}

1. Bennett AK, Mills SE, Wick MR. Salivary-type neoplasms of the breast and lung. Semin Diagn Pathol 2003;20:279-304.

2. Macchiarini P. Primary tracheal tumours. Lancet Oncol 2006; 7:83-91.

3. Stevic R, Milenkovic B. Tracheobronchial tumors. J Thorac Dis 20 2016;8:3401-13.

4. Qin BD, Jiao XD, Liu K, et al. Clinical, pathological and treatment factors associated with the survival of patients with primary pulmonary salivary gland-type tumors. Lung Cancer 2018;126:174-81.

5. Hogerle BA, Lasitschka F, Muley T, et al. Primary adenoid cystic carcinoma of the trachea: clinical outcome of 38 patients after interdisciplinary treatment in a single institution. Radiat Oncol 2019;14:117.

6. Yang CJ, Shah SA, Ramakrishnan D, et al. Impact of Positive Margins and Radiation After Tracheal Adenoid Cystic Carcinoma Resection on Survival. Ann Thorac Surg 2020;109:1026-32.

7. Ngo AV, Walker CM, Chung JH, et al. Tumors and tumorlike conditions of the large airways. AJR Am J Roentgenol 2013;201:301-13.

8. Calzada AP, Miller M, Lai CK, et al. Adenoid cystic carcinoma of the airway: a 30-year review at one institution. Am J Otolaryngol 2012;33:226-31.
9. Gaissert HA, Grillo HC, Shadmehr MB, et al. Longterm survival after resection of primary adenoid cystic and squamous cell carcinoma of the trachea and carina. Ann Thorac Surg 2004;78:1889-96; discussion 1896-7.

10. Luo M, Duan C, Qiu J, et al. Diagnostic Value of Multidetector CT and Its Multiplanar Reformation, Volume Rendering and Virtual Bronchoscopy Postprocessing Techniques for Primary Trachea and Main Bronchus Tumors. PLoS One 2015;10:e0137329.

11. Goldsher D, Litt AW, Pinto RS, et al. Dural "tail" associated with meningiomas on Gd-DTPA-enhanced MR images: characteristics, differential diagnostic value, and possible implications for treatment. Radiology 1990;176:447-50.

12. Maziak DE, Todd TR, Keshavjee SH, et al. Adenoid cystic carcinoma of the airway: thirty-two-year experience. J Thorac Cardiovasc Surg 1996;112:1522-31.

13. Ning Y, He W, Bian D, et al. Tracheo-bronchial adenoid cystic carcinoma: A retrospective study. Asia Pac J Clin Oncol 2019;15:244-9.

14. Je HU, Song SY, Kim DK, et al. A 10-year clinical outcome of radiotherapy as an adjuvant or definitive treatment for primary tracheal adenoid cystic carcinoma. Radiat Oncol 2017;12:196.

15. Ratto GB, Alloisio A, Costa R, et al. Primary peripheral adenoid cystic carcinoma of the lung. A case report. Acta Chir Belg 2003;103:414-5.

16. Zhao Y, Zhao H, Fan L, et al. Adenoid cystic carcinoma in the bronchus behaves more aggressively than its tracheal counterpart. Ann Thorac Surg 2013;96:1998-2004.

17. Jeong SY, Lee KS, Han J, et al. Integrated PET/CT of salivary gland type carcinoma of the lung in 12 patients. AJR Am J Roentgenol 2007;189:1407-13.

18. Maziak DE. Biology of Adenoid Cystic Carcinoma of the Tracheobronchial Tree and Principles of Management. Thorac Surg Clin 2018;28:145-8.

19. Bhattacharyya $\mathrm{N}$. Contemporary staging and prognosis for primary tracheal malignancies: a population-based analysis. Otolaryngol Head Neck Surg 2004;131:639-42.

(English Language Editors: B. Draper and J. Chapnick)

Cite this article as: Wang SC, Yin LK, Zhang Y, Xue LM, Ye JD, Tao GY, Yu H, Qiang JW. Contrast-enhanced CT longitudinal tail sign as a marker of positive resection margins in adenoid cystic carcinoma of the central airway. J Thorac Dis 2021;13(5):2803-2811. doi: 10.21037/jtd-20-2929 